\title{
Numerical Model Reduction with Error Estimation for Computational Homogenization of Nonlinear Poroelasticity
}

\author{
Fredrik Ekre $^{\dagger *}$, Fredrik Larsson ${ }^{\dagger}$, Kenneth Runesson $^{\dagger}$, and Ralf Jänicke ${ }^{\dagger}$ \\ * Division of Material and Computational Mechanics, Dept. of Industrial Materials Science \\ Chalmers University of Technology \\ Hörsalsvägen 7A, 41296 Gothenburg, Sweden \\ e-mail: \{fredrik.ekre\}\{fredrik.larsson\}\{kenneth.runesson\}\{ralf.janicke\}@chalmers.se
}

\begin{abstract}
In order to model the effective mechanical behavior of fluid saturated porous rock with heterogeneous properties, computational homogenization can be adopted. One standard approach is the so-called "finite element squared" $\left(\mathrm{FE}^{2}\right)$ procedure, where a new boundary value problem for the coupled porous media problem is defined on a Representative Volume Element (RVE) in each quadrature point of the (macroscale) mesh. In order to obtain the effective response of the material the RVE problem is solved, and the results are averaged whereby the RVE computation replaces the otherwise needed macroscale constitutive relation of empirical character. It is known that the $\mathrm{FE}^{2}$ strategy can be computationally intractable and it is therefore of interest to reduce the cost of solving the individual RVE problems by introducing a reduced basis, here denoted Numerical Model Reduction (NMR).

Jänicke et al. [1] considered homogenization of quasi-static linear poroelasticity with application to seismic attenuation. It was demonstrated that the apparent macroscale properties pertain to viscoelasticity. Proper Orthogonal Decomposition (POD) was used to find a set of pressure modes that defined the reduced problem, and the efficiency of the procedure was demonstrated. The richness of the reduced basis will determine the accuracy of the solution, which calls for error control. Ekre et al. [2] developed an a posteriori error estimator for estimating the error stemming from the application of the reduced basis, in terms of an energy norm as well as for arbitrary quantities of interest. The error estimator utilizes the linearity of the problem with guaranteed error bounds as a result.

In this contribution, we further develop the previous work and consider the non-linear problem with deformation dependent permability. We use the reduced model from $[1,2]$ and adopt POD to construct the reduced basis and define the reduced problem. The major novelty is new error estimator for estimating the NMR error originating from the reduced basis. Due to the non-linearity it is not possible to employ the same strategy for the estimator and find guaranteed bounds. Instead we attempt to derive an explicit, fully computable, and low cost, estimator that approximates the error.

The performance of the estimator is demonstrated with a few numerical examples concerning an heterogeneous RVE structure in three dimensions. In particular, the effect of the loading amplitude, and thus the magnitude of the non-linearity, on the robustness of the estimator is investigated.
\end{abstract}

\section{REFERENCES}

[1] R. Jänicke, F. Larsson, K. Runesson, and H. Steeb. Numerical identification of a viscoelastic substitute model for heterogeneous poroelastic media by a reduced order homogenization approach. Comput. Methods Appl. Mech. Engrg. (2020), DOI: 10.1016/j.cma.2015.09.024.

[2] F. Ekre, F. Larsson, K. Runesson, and R. Jänicke. A Posteriori Error Estimation for Numerical Model Reduction in Computational Homogenization of Porous Media. International Journal for Numerical Methods in Engineering (2020), DOI: 10.1002/nme.6504. 